\title{
Neuromuscular electrical stimulation for mobility support of elderly
}

\author{
Winfried Mayr \\ Center of Medical Physics and Biomedical Engineering, Medical University \\ of Vienna, Austria.
}

\begin{abstract}
The stimulator for neuromuscular electrical stimulation for mobility support of elderly is not very complicated, but for application within "MOBIL" we have some additional demands to fulfill. First we have specific safety issues for this user group. A powerful compliance management system is crucial not only to guide daily application, but for creating hard data for the scientific outcome. We also need to assure easy handling of the stimulator, because the subjects are generally not able to cope with too difficult and complex motor skills. So, we developed five generations of stimulators and optimizing solutions after field tests. We are already planning the sixth generation with wireless control of the stimulation units by the central main handheld control unit. In a prototype, we have implemented a newly available high capacity memory, a breakthrough in "compliance data storage" as they offer the necessary high storage capacity and fast data handling for an affordable prize. The circuit also contains a 3D accelerometer sensor which acts as a further important safety features: if the control unit drops, this event is detected automatically by the sensor and activates an emergency switch-off that disables the stimulation to avoid associated risks. Further, we have implemented a hardware emergence shutdown and other safety measures. Finally, in the last example muscle torque measurements are referenced with compliance data. In the study normalized maximum voluntary contraction (MVC) and maximum stimulation induced contraction (MSC) were assessed in regular check-ups along the training period. With additional consideration of adjusted stimulation intensity for training out of the compliance data records we are able to estimate the induced contraction strength, which turned out to amount in average $11 \%$ of MVC. This value may seem on a first sight rather low, and ought to be considered in relation to the results at the end of the training period. Therefore the correlation between normalized MVC and normalized MSC was calculated. It is obvious that MVC can increase to strongly variable extent ( 3 to $65 \%$ ), but in few cases also decrease (-4 to 15 $\%$ ) over the study period. The correlation suggests that an increase of roughly $1 \%$ of normalized MSC can lead to an increase of about $10 \%$ in MVC in the given training conditions. Overall, we can say that we have a stimulator that has turned out to work sufficiently. The most important feature is the integrated compliance recording because this is very useful for interpretation of the study outcome. The electrical stimulation training has shown that even with relatively small induced contraction intensity we still get some increase in the achievable voluntary extension torque.

Key words: aging, skeletal muscle, neuromuscular electrical stimulation
\end{abstract}

Eur J Transl Myol - Basic Appl Myol 2015; 25 (4): 263-268

Good morning, everybody! I am glad to see so many of you here in Vienna again. I start with an overview of the working group, which is hopeless to display completely on one slide, so I have some dots there for "etc." in the list (Fig. 1).

We have a quite interdisciplinary discipline team with engineering, which is our part, with sports sciences and with biological sciences and today it is my role to give some engineering insight into the stimulator development. The stimulator per se is not very complicated, but for application within "MOBIL" we have some additional demands to fulfill.

First we have specific safety issues for this user group. A powerful compliance management system is crucial not only to guide daily application, but especially for creating hard data for the scientific outcome. ${ }^{1,2}$ We also need to assure easy handling of the stimulator, because the subjects are generally not able to cope with too difficult and complex motor skills. 


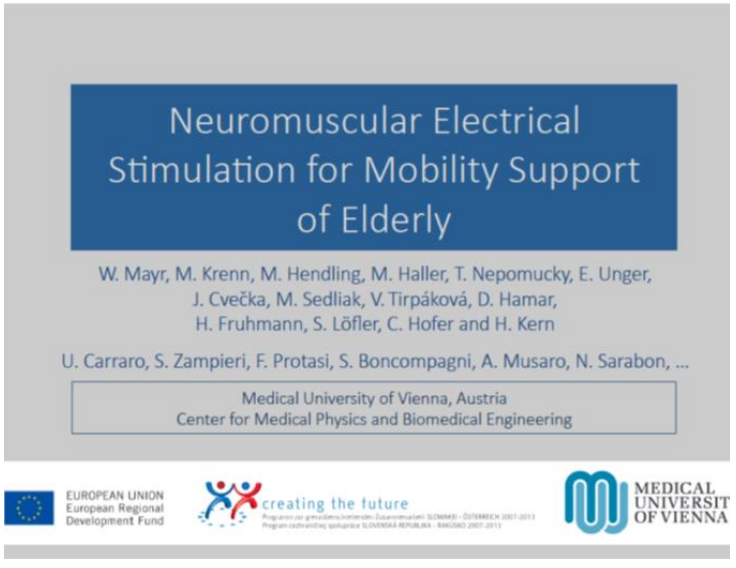

Fig 1. Project, the engineering contribution in a multidisciplinary consortium

So, we developed several generations of stimulators and optimized solutions after field tests. We see five development steps on this slide, number 5 is the actual one and we are already planning the sixth generation with wireless control of the stimulation units by the central main handheld control units (Fig. 2). On this slide we see a magnified view of the actual central unit with quite simple user interface and two stimulation units, which are the active units to be placed on each anterior thigh. Inside we have more complex technical features that might be of interest. Basically we try to rely on standard technology as far as possible.

You might notice the wireless charging system, like quite common for mobile phones or tablets; we have implemented this option, based on the upcoming QIstandard already in the electronic circuit. A USB connection for data transfer makes interaction with a standard computer easier, as a prototype we also have a Bluetooth link, substituting this wired interface, in operation.

We have implemented a newly available high capacity SD standard memory cards and those provide a breakthrough in "compliance data storage" as they offer the necessary high storage capacity and fast data handling now for an affordable prize. The circuit also contains a 3D accelerometer sensor which acts as a further important safety features: if the control unit drops, this event is detected automatically by the sensor and activates an emergency switch-off and disables the stimulation to avoid associated risks.

On the other hand we have implemented a hardware emergence shutdown, which is also crucial, because various complications in a software controlled complex electronic system may occur and demand intervention. Malfunction of the control program may require disconnection of power supply for immediate reliable deactivation. An overview with all mechanical components of the central unit shows the case with front and rear part, the main printed port (PCB), switches, display battery and connectors. The case is a $3 D$ print in acrylic resin

\section{Stimulation system}

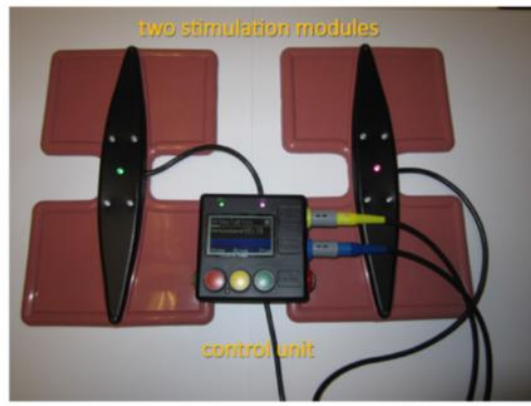

Fig 2. Version 5 of the stimulation system as applied in the MOBIL study

after 3D design using appropriate computer software. The miniaturized electronic circuit is quite complex and includes connections to control switches, display and connectors.

The electrodes are embedded in a casted silicone carrier. Before casting a fiberglass reinforcements element with solid fixation means for the electronics is placed in the cast. The stimulator PCB carries its own micro controller and is able to stimulate in a stand-alone mode. We need to produce high voltage out of low voltage battery supply on this peripheral electronic circuit. Power supply currently is provided by the battery in the central unit via cable connection, this will be substituted by peripheral rechargeable batteries in the stimulation units as soon as wireless control will be implemented. Also the electronic circuit of the stimulation units contains 3D accelerometer sensors, which are nowadays available as sensitive robust and cheap components, as they are produced for millions of mobile phones and tablet computers, where they detect position and orientation of the device in relation to the gravity vector. We rely on the same principle to monitor if the patient device is appropriately placed prior to and during operation. It tells us if he is in seating position, like he should be during the entire training. Altering this position, e.g. standing up leads to automated termination of stimulation. On the other hand these sensors are so sensitive that we can also sense mechanical muscle twitches and even detect thresholds for stimulated muscle reactions.

Audience question: Sorry, this monitor is sensing the contractions of the muscle?

Mayr: Yes, it has a high dynamic range so it can sense very low changes in sensor position in relation to the gravity vector in $3 \mathrm{D}$, on the other hand it certainly can detect movements the body surface above the belly of a contracting muscle, where it is mounted in close contact and measures an acceleration vector orthogonal to the surface.

The sensor is mounted to the printed board detects movement like a modern mobile phone that can detect movement and orientation in space and 


\title{
Stimulation parameter
}

\author{
Technology: \\ voltage-controlled pulse \\ Pulse shape: \\ charge-balanced, rectangular, biphasic
}

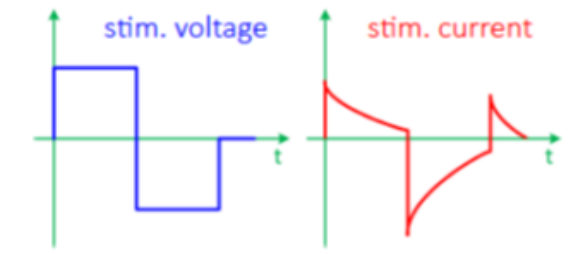

\begin{tabular}{|c|c|c|}
\hline Stimulation parameter & Range & Resolution \\
\hline \multicolumn{3}{|l|}{ Amplitude control: } \\
\hline stimulation voltage & $U_{\text {BAT }}-45.65 \mathrm{~V}$ & $0.357 \mathrm{~V}$ \\
\hline \multicolumn{3}{|l|}{ Burst element: } \\
\hline stimulation pulse rate $\left(1 / T_{p E R}\right)$ & $0.01-200 \mathrm{pps}$ & $0.04 \mathrm{pps}$ (at $200 \mathrm{pps}$ ) \\
\hline number of pulses & $0-2^{32}$ & 1 \\
\hline burst element duration $\left(T_{\mathrm{BE}}\right)$ & $0.005-7200 \mathrm{~s}$ & $1 \mathrm{~ms}$ \\
\hline \multicolumn{3}{|l|}{ Pulse timing: } \\
\hline positive pulse width $\left(T_{A}\right)$ & 0 or $50-1000 \mu \mathrm{s}$ & $1 \mu \mathrm{s}$ \\
\hline inter-pulse time $\left(T_{B}\right)$ & 0 or $50-65535 \mu \mathrm{s}$ & $1 \mu \mathrm{s}$ \\
\hline negative pulse width $\left(T_{C}\right)$ & 0 or $50-1000 \mu \mathrm{s}$ & $1 \mu \mathrm{s}$ \\
\hline delay of next pulse let $\left(T_{D}\right)$ & 0 or $50-65535 \mu \mathrm{s}$ & $1 \mu \mathrm{s}$ \\
\hline n-lets & $1-16$ & 1 \\
\hline
\end{tabular}

\section{Fig 3 . Stimulation pattern and impulse ranges}

adapt the display orientation accordingly, but also is able to, for example, count impacts of steps for certain apps. We also have integrated an impedance measurement circuit, which is another important safety feature to check the electrode quality and correct placement.

Audience question: Can you explain why it is necessary for safety?

Mayr: Basically skin damage could be induced during stimulation, if part of conductivity through the electrode tissue interphase is lost. In case of failure, like an asymmetric stimulus occurring or exceeding of a certain amount of charge per impulse, so-called charge injection limits, the recharge phase of a stimulus cannot compensate charge flow entirely and electrochemical reaction get irreversible with potential tissue damage. Part of these chemical reactions are buffered within a gel or a wetted sponge layer between electrode and skin, but with increase of duration of training or increase of current intensity damaging reactions can reach the skin tissue. This is somehow a permanent risky and we need to be very careful. So we rely on the capacitors to force charge balance, but many stimulators are on the market that just control charge balance by a control algorithm that like all technical solutions can fail and induce tissue damage.

We apply biphasic rectangular stimulation pulses with amplitudes of up to $+/-45 \mathrm{~V}$. The voltage trace is controlled $(\mathrm{CV})$, the current trace follows in dependence of the electrode-tissue impedance. The stimulator can deliver up to 200 pulses per second (pps) or stimulate with up to $200 \mathrm{~Hz} .200 \mathrm{~Hz}$ can e.g. be used for blocking nerve conductivity for action potentials. We can freely adapt amplitude, impulse width and frequency in dependence of the application. Normally, we use impulses with a soft start ramp (Fig. 3).

A very important special feature is the integrated compliance management system, which is extremely important for guaranteeing validity of study data. E. g. we can follow all adjustments a patient has made during his entire application of the stimulator. The recorded data are stored on SD card and can be transferred to the computer via USB data link for analysis. This helps tremendously for the interpretation of scientific outcome. I will give you a few examples based on selectively extracted data. In principle we store a huge amount of raw data that are of course in this basic form useless for the supervising physician, who wants to interpret them and use them for follow-up. A diploma thesis, just recently finalized by Michaela Hendling, addressed this topic and aimed in systematic extraction and visualization of useful data. The system stores raw data on the following parameters:

- Date, time of training

- Complete stimulation program

- Recording of stimulation voltage shape and current shape of single impulses.

- Recording of actual stimulation amplitude adjustments over time

- Accelerometer vector data over time

- Any occurring error 


\section{Results of 10 weeks of training}

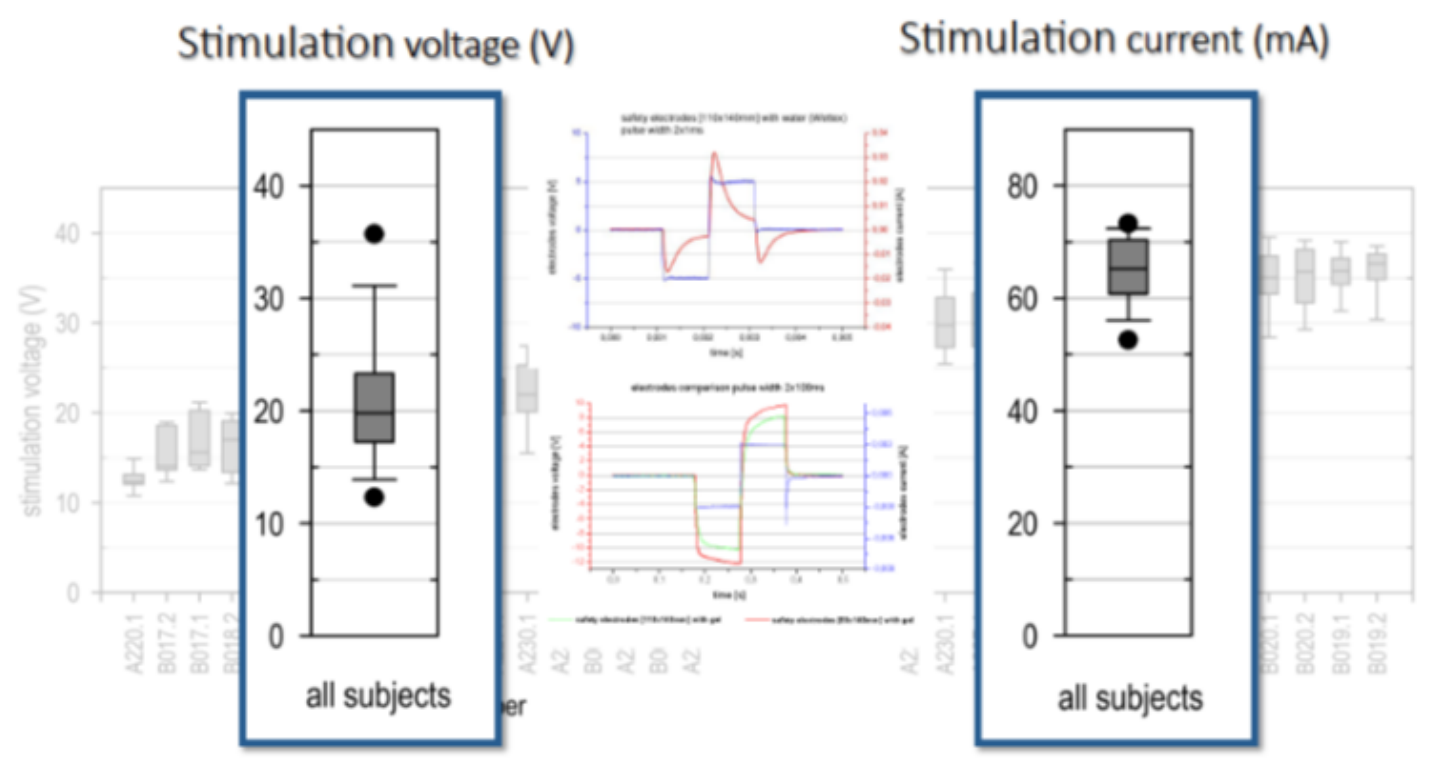

Fig 4. Average voltage and current variation in all participants along the entire study and comparison of impulse shapes in voltage and current control (VC and CC) mode

In this example the adjustment of voltage amplitude over time is shown as manually adjusted by the patient as maximum still sensibly comfortable maximum amplitude. A follow-up of voltage and current amplitude is shown. These are average values of a series of sessions, in reality we see that the patients starts with lower intensity and slowly increases the voltage up to $28 \mathrm{~V}$ within several minutes. This is a clear example for the helpfulness of this detailed data assessment for support of valid interpretations. We see that it can take half a training session till constant training conditions are reached, which is understandable but needs to be considered in the interpretation. In another example we see a training protocol over 10 weeks in total, starting with $2 \times 3$ training series of 6 min per week, increased to $2 \times 3$ training sessions of $10 \mathrm{~min}$ per week for week 3 to 6 and finally increased to $3 \times 3$ 10 minute sessions for the remaining observation period. In the compliance data we can see how the patient has adjusted the voltage level along 48 trainings how this level varies quite strongly. The associated current level varies much less. This is quite interesting and tells us that the daily application of the electrodes did not provide constant contact resistance of the electrode tissue interface, but variation in wetting the contact area and/or adjustment of contact pressure had been far from constant. The patient adjusted the voltage amplitude based on sensible perception and ended up with pretty much constant current amplitude values. If we compare the average set voltage amplitude levels of all patients we can see that there were patients, who were very sensitive and kept amplitudes low, and there was one particular patient, who had always applied a huge intensity levels close to the maximum the stimulator is able to deliver. It turned out that he is a retired power electrician and is well adapted to sensations induced by electrical currents. If we compare the box plots of voltage and current levels integrating all training sessions of all study participants we see a big variation of adjusted voltage and much less variation in the resulting current stimulation (Fig. 4). This of course leads to the basic question: why don't we apply constant current stimuli? In this form the question is too simple, which becomes obvious, if we look at the impulse shape of controlled voltage and controlled current mode. In addition to control of current level to sensibly tolerable threshold there are non-negligible influences if impulse shape on efficiency of nerve fiber recruitment as well as on sensible comfort, and with this indirectly on adjustable intensity maximum We have done additional systematic studies on these phenomena, but today due to time constrains I cannot report on this.

Finally, in the last example muscle torque measurements are referenced with compliance data. In the study normalized maximum voluntary contraction (MVC) and maximum stimulation induced contraction (MSC) were assessed in regular 


\section{Induced muscle torque}

Normalized torque difference between pre- and post training

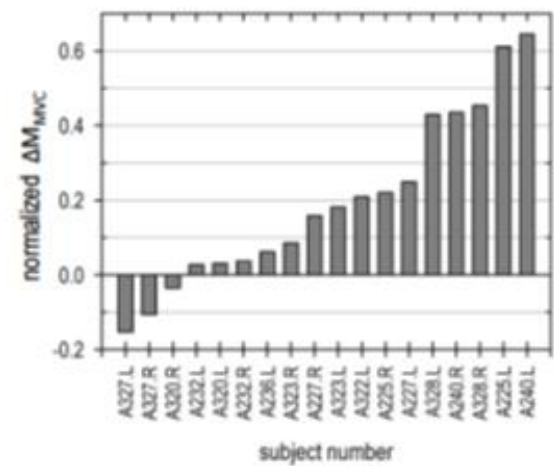

Statistically significant increase in muscle torque (Paired t-test: $p=0.009$ )
Correlation between normalized induced torque and pre-post torque difference

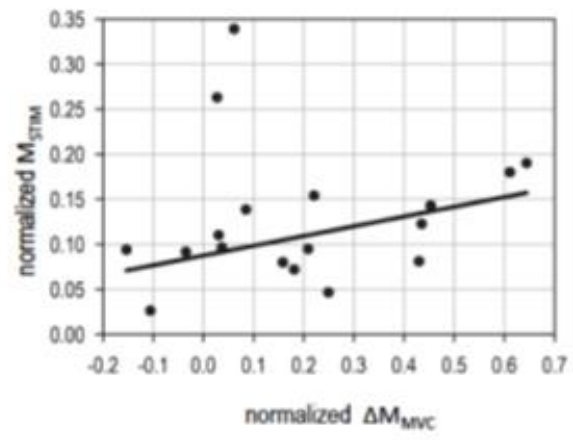

Statistically significant increase in muscle torque (Paired t-test: $p=0.009$ )

Fig 5. MVC development under FES training and relation of stimulation induced and voluntary maximum knee extension torque.

check-ups along the training period. With additional consideration of adjusted stimulation intensity for training out of the compliance data records we are able to estimate the induced contraction strength, which turned out to amount in average $11 \%$ of MVC. This value may seem on a first sight rather low, and ought to be considered in relation to the results at the end of the training period. Therefore the correlation between normalized MVC and normalized MSC was calculated. It is obvious that MVC can increase to strongly variable extent (3 to $65 \%$ ), but in few cases also decrease (-4 to $15 \%)$ over the study period. The correlation suggests that an increase of roughly $1 \%$ of normalized MSC can lead to an increase of about $10 \%$ in MVC in the given training conditions (Fig. 5). Overall, we can say that we have a stimulator that has turned out to work sufficiently. The most important feature is the integrated compliance recording because this is very useful for interpretation of the study outcome.

The electrical stimulation training has shown in its results that even with relatively small induced contraction intensity we still get some increase in the achievable voluntary extension torque. I think we will hear more on medical and biological aspects in the next presentations by our specialized colleagues.

Finally I want to thank all involved contributors. We have a large group of students involved, preparing their theses on this subject (Fig. 6). On the whole, our work is an engineering contribution within the work of a vastly interdisciplinary team and I look very much forward to the coming presentations to learn more on biological and clinical aspects.
Discussion at the end of the Presentation of Prof. Mayr Carraro: Are there any questions?

Kern: I think it is important to note what he has done for future projects. We have to detect what is the compliance of the patient, what is the amount of stimulation, what is the outcome of the relation of maximum stimulated to maximum voluntary contraction and therefore how many percent of muscle contraction we induce by this technique. We will hear from Sandra Zampieri the results from the leg press training with high intensity and electrical stimulation with moderate intensity. It is a little bit different but related, because it is not so different from the functional outcome. The Bratislava group will talk about the training program from the seniors in relation to the contraction, the intensity of contraction and the maximum voluntary contraction, of which they have a precise protocol.

Data collection is also very important to see what patients are doing at home. If they are motivated, they adjust the voltage and the intensity of stimulation, and they increase the intensity themselves. It is very important to collect these data to compare what the patient did versus the physiological outcomes.

Audience question: I don't understand how you correct the intensity for different patients like obese patients or patients with little muscle mass. The resistance in obese patients will be a bit bigger I assume. Further, patients with a lot of leg hair need to be shaved? 


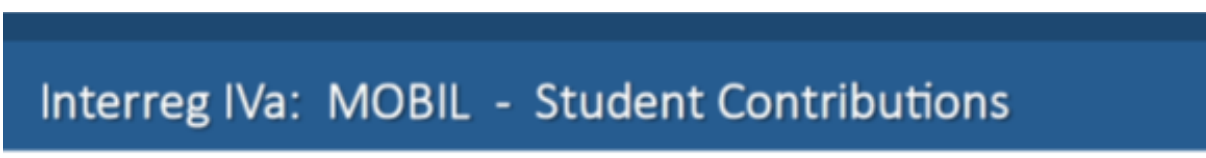

\title{
Coordination of all developments and study engineering tasks:
Matthias Krenn - PhD Thesis
}

\author{
Hardware developments: \\ - Michael Haller-MSc Thesis \\ - Fabian Padrta - BSC Thesis \\ - Ing. Ewald Unger \\ - Sing Yeung
}

Electrode developments:

- Elentari Nepomucky-MSc Thesis

- Cristina Hanc-MSc Thesis

- Andreas Ortt-BSc Thesis

Software and compliance:

- Michaela Hendling - MSc Thesis

- Florian Guggenberger - MSc Thesis

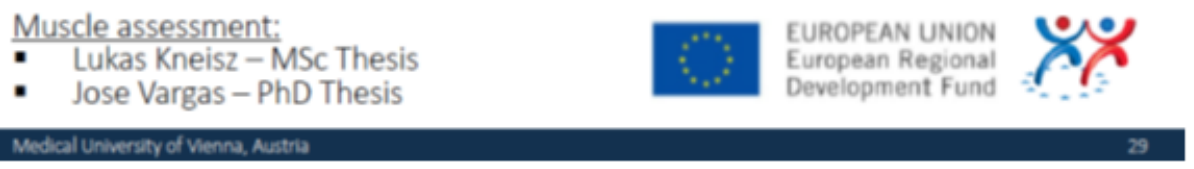

\section{Fig 6. Contributions of PhD Students to MOBIL}

Mayr: This is not so critical but we have assessment sessions in the beginning and later on, where we measure isometric characteristic and adapt the stimulation. Therefore, we have some kind of calibration. The variability of the currents was not that big. We can check on a measurement scale and see how to the reactions changes at certain levels. It is not very exact, but it can be integrated in the treatment of the patients.

Carraro: I understood that the load in the muscle is at a relatively low level, $10 \%$ of what the patient can do by himself. I am asking if you put 2 to 5 kilos on that leg, they will do the same contraction, the same effort, the same metabolic equivalent of activity? Could this be an additional variable to take into consideration? Could it be monitored?

Kern: We have done it.

Mayr: The precise effect of intensity is depending on the patient's sensation. They do what still feels comfortable.

Kern: Somebody did the stimulation without weights and then we added ankle weights to increase the contraction. You will hear more of it today.

Mayr: It is important to note that we have this absolute maximum level, where the patient starts to feel uncomfortable. All the rest is influenced by training factors like weights, contraction time, number of repetitions and so on. We had rare examples like our colleague and physiotherapist Andreas Kainz, who was able to tolerate stimulation intensities eliciting contractions close to MVC level, but such examples are rare exceptions.

Kern: One final thing, in this approach of electrostimulation it is not so easy to detect the true force of the contraction because if you stimulate the quadriceps with large electrodes, there is concomitantly some co-contraction of the hamstring muscle that compensate part of the extension torque by a flexion component. It depends on the intensity of the current.

Carraro: My question was: it was possible to monitor how much load you put on the leg or did you have to write it down on paper?

Mayr: We could develop technical solutions for that but we did not yet.

Carraro: Because if you might lower the stimulation parameters and obtain a better effect, you should be able to monitor it.

Mayr: We could think about it. It is an interesting question.

\section{Address for correspondence}

Winfried Mayr, Center of Medical Physics and Biomedical Engineering, Medical University of Vienna, Austria.

Phone: + 4400431404001982

E-mail: winfried.mayr@meduniwien.ac.at

\section{References}

1. Zampieri S, Pietrangelo $L$, Loefler $S$, et al. Lifelong Physical Exercise Delays AgeAssociated Skeletal Muscle Decline. J Gerontol A Biol Sci Med Sci 2015;70:163-3.

2. Kern $H$, Barberi L, Löfler $S$, et al. Electrical stimulation counteracts muscle decline in seniors. Front Aging Neurosci. 2014 Jul 24;6:189. doi: 10.3389/fnagi.2014.00189. eCollection 2014. 